\title{
Sliding Mode Control and Active Disturbance Rejection Control to the Stabilization of One-Dimensional Schrödinger Equation subject to Boundary Control Matched Disturbance*
}

\author{
Bao-Zhu Guo ${ }^{a, b \dagger}$ and Jun-Jun Liu ${ }^{c}$ \\ ${ }^{a}$ Academy of Mathematics and Systems Science \\ Academia Sinica, Beijing 100190, China \\ ${ }^{b}$ School of Computational and Applied Mathematics \\ University of the Witwatersrand, Wits 2050, Johannesburg, South Africa \\ ${ }^{c}$ School of Mathematical Sciences \\ Beijing Institute of Technology, Beijing 100081, China
}

January 26, 2013

\begin{abstract}
In this paper, we are concerned with the boundary stabilization of a one-dimensional antistable Schrödinger equation subject to boundary control matched disturbance. We use both the sliding mode control (SMC) and the active disturbance rejection control (ADRC) to deal with the disturbance. By the SMC approach, the disturbance is supposed to be bounded only. The existence and uniqueness of the solution for the closed-loop system is proved and the "reaching condition" is obtained. Considering the SMC usually requires the large control gain and may exhibit chattering behavior, we develop the ADRC to attenuate the disturbance for which the derivative is also supposed to be bounded. Compared with the SMC, the advantage of the ADRC is not only using the continuous control, but also giving an online estimation of the disturbance. It is shown that the resulting closed-loop system can reach any arbitrary given vicinity of zero as time goes to infinity and high gain tuning parameter goes to zero.
\end{abstract}

Keywords: Schrödinger equation, sliding mode control, active disturbance rejection control, stability, boundary control, disturbance rejection.

AMS subject classifications: 35J10, 93C20, 93C25.

${ }^{*}$ This work was supported by the National Natural Science Foundation of China, the National Basic Research Program of China (2011CB808002), and the National Research Foundation of South Africa.

${ }^{\dagger}$ Corresponding author. Email: bzguo@iss.ac.cn. 


\section{Introduction}

For a system described by partial differential equations (PDEs), the boundary control dominates the research tread in the past two decades, see $[3,6,17,20]$ and the references therein. Traditionally, the system is controlled in the ideal operational environment with exact mathematical model and no internal and external disturbances. This can be found in many researches for the stabilization of infinite-dimensional systems described by Schrödinger, wave and flexible beam equations ([3, 20, 21]).

Basically speaking, two different type of control methods can be used to stabilize the PDEs without disturbance. The collocated control design is based on the passive principle that makes the closed-loop system dissipative and hence stable at least in the sense of Lyapunov ([3]). The non-collocated method is systematically applied, due to the introduction of backstepping method to PDEs in the last few years ([17], see also [6]), to stabilize some unstable or even anti-stable wave and heat equations $([15,17,27])$.

However, "if there is no uncertainty in the system, the control, or the environment, feedback control is largely unnecessary" ([2]). When the external disturbances enter the system from boundary or the internal of the spatial domain, the new approach is needed to deal with the uncertainties. The adaptive control method is powerful in dealing with the systems with the unknown parameters $([7,8,16])$. The general method to reject the disturbance is the sliding mode control (SMC) method $([1,4,5,23,26])$. In [23], based on the semigroup theory, the SMC is used to deal with a class of abstract infinite-dimensional systems where the control and disturbance are all assumed to be bounded (mainly in distributed control). The boundary stabilization for a one-dimensional heat equation with boundary disturbance is studied in [5], where the SMC is designed for the first-order PDEs obtained through an integral transformation on the heat equation (which is second order in spatial variable). Very recently, the sliding mode boundary stabilizer is designed for a onedimensional unstable heat and wave equation in [4] and [9] respectively. Another powerful method in dealing with the disturbance is the active disturbance rejection control (ADRC) method. The ADRC, as an unconventional design strategy, was first proposed by Han in 1990s ([13]). It has been now acknowledged to be an effective control strategy for lumped parameter systems in the absence of proper models and in the presence of model uncertainty. The numerous applications have been carried out in the last decade (see e.g., [28]). Its convergence has been proved for lumped parameter systems in [10]. Very recently, it has been successfully applied to the attenuation of disturbance for a one-dimensional anti-stable wave equation in [9]. Other method in dealing with uncertainty includes the Lyapunov function based method, see [11, 25] and the references therein.

Motivated mainly by [9] and [14], we are concerned with, in this paper, the stabilization of a one-dimensional Schrödinger equation which is suffered from the unknown external disturbance on the input boundary by both the SMC approach and the ADRC approach, respectively. 
The system that we are concerned with is governed by the following PDEs:

$$
\left\{\begin{array}{l}
u_{t}(x, t)=-j u_{x x}(x, t), x \in(0,1), t>0 \\
u_{x}(0, t)=-j q u(0, t), q>0, t \geqslant 0 \\
u_{x}(1, t)=U(t)+d(t), t \geqslant 0
\end{array}\right.
$$

where $u$ is the complex-valued state, $j$ is the imaginary unit, $U$ is the control input. The unknown disturbance $d$ is supposed to be uniformly bounded measurable, that is, $|d(t)| \leqslant M_{0}$ for some $M_{0}>0$ and all $t \geqslant 0$. The system represents an anti-stable distributed parameter system: all eigenvalues of the free system (with no control and disturbance) are located on the right-half complex plane.

The main contribution of this paper is to apply both the SMC and the ADRC approaches to reject and attenuate respectively the disturbance in the stabilization of the system (1.1). The distinguish feature of this problem that is contrast to the wave and beam equations is that the state variable is complex valued. This gives rise to some problems in terms of mathematical rigorousness. For instance, the sliding modes are actually two by its real and imaginary parts while that of beam or wave is only one in the real number field space $([9])$.

The rest of the paper is organized as follows. Section 2 is devoted to the disturbance rejection by the SMC approach. The sliding mode control is designed and the existence and uniqueness of solution of the closed-loop system are proved. The finite time "reaching condition" is presented rigorously. In Section 3, we use the ADRC approach to attenuate the disturbance by designing a high gain estimator to estimate the disturbance. After canceling the disturbance by the approximated one, we design the state feedback controller. The closed-loop system is shown to attend any arbitrary given vicinity of zero as the time goes to infinity and the gain tuning parameter tends to zero. Some concluding remarks are presented in Section 4.

\section{Sliding mode control approach}

We consider system $(1.1)$ in the state space $\mathcal{H}=L^{2}(0,1)$. Following [27], we introduce a transformation:

$$
w(x, t)=u(x, t)-\int_{0}^{x} k(x, y) u(y, t) d y
$$

where the gain kernel $k$ satisfies the following PDE:

$$
\left\{\begin{array}{l}
k_{x x}(x, y)-k_{y y}(x, y)=c j k(x, y), c>0 \\
k_{y}(x, 0)+j q k(x, 0)=0 \\
k(x, x)=-\frac{c j}{2} x-j q
\end{array}\right.
$$

The existence of solution to problem (2.2) can be proved by transforming it into an integral equation using the variable change $\xi=x+y, \eta=x-y$, which is Lemma 2.1 below (see [27]).

Lemma 2.1 The problem (2.2) admits a unique solution which is twice continuously differentiable in $0 \leqslant y \leqslant x$. 
Proof. We introduce new variables

$$
\xi=x+y, \eta=x-y
$$

and denote

$$
G(\xi, \eta)=k(x, y)=k\left(\frac{\xi+\eta}{2}, \frac{\xi-\eta}{2}\right) .
$$

To derive equation in terms of these new variables, we compute

$$
\begin{array}{ll}
k_{x}(x, y)= & G_{\xi}(\xi, \eta)+G_{\eta}(\xi, \eta), \\
k_{y}(x, y)= & G_{\xi}(\xi, \eta)-G_{\eta}(\xi, \eta), \\
k_{x x}(x, y)= & G_{\xi \xi}(\xi, \eta)+2 G_{\xi \eta}(\xi, \eta)+G_{\eta \eta}(\xi, \eta), \\
k_{y y}(x, y)= & G_{\xi \xi}(\xi, \eta)-2 G_{\xi \eta}(\xi, \eta)+G_{\eta \eta}(\xi, \eta), \\
k(x, x)= & G(\xi, 0), k_{y}(x, 0)=G_{\xi}(\xi, \xi)-G_{\eta}(\xi, \xi), k(x, 0)=G(\xi, \xi) .
\end{array}
$$

Substitute these derivatives into (2.2) to yield

$$
G_{\xi \eta}(\xi, \eta)=\frac{c j}{4} G(\xi, \eta), 0 \leqslant \eta \leqslant \xi \leqslant 2
$$

with the boundary conditions:

$$
\left\{\begin{array}{l}
G_{\xi}(\xi, \xi)-G_{\eta}(\xi, \xi)+j q G(\xi, \xi)=0 \quad 0 \leqslant \xi \leqslant 2 \\
G(\xi, 0)=-\frac{\xi c j}{4}-j q, 0 \leqslant \xi \leqslant 2 . \\
G_{\xi}(\xi, 0)=-\frac{c j}{4}
\end{array}\right.
$$

Integrate $(2.5)$ over $[0, \eta]$ with respect to $\eta$, and use the third condition of (2.6), to obtain

$$
G_{\xi}(\xi, \eta)=-\frac{c j}{4}+\frac{c j}{4} \int_{0}^{\eta} G(\xi, s) d s .
$$

Integrate $(2.7)$ over $[\eta, \xi]$ with respect to $\xi$ to give

$$
G(\xi, \eta)=G(\eta, \eta)-\frac{c j}{4}(\xi-\eta)+\frac{c j}{4} \int_{\eta}^{\xi} \int_{0}^{\eta} G(\tau, s) d s d \tau
$$

To find $G(\eta, \eta)$, we use (2.6) to write

$$
\frac{d}{d \xi} G(\xi, \xi)=G_{\xi}(\xi, \xi)+G_{\eta}(\xi, \xi)=2 G_{\xi}(\xi, \xi)+j q G(\xi, \xi) .
$$

Using (2.7) with $\eta=\xi$, we can write (2.9) in the form of differential equation for $G(\xi, \xi)$ :

$$
\frac{d}{d \xi} G(\xi, \xi)=-\frac{c j}{2}+\frac{c j}{2} \int_{0}^{\xi} G(\xi, s) d s+j q G(\xi, \xi) .
$$

Integrate (2.10) by making use of the variation of constants formula to obtain

$$
G(\xi, \xi)=-j q e^{j q \xi}-\frac{c j}{2} \int_{0}^{\xi} e^{j q(\xi-\tau)} d \tau+\frac{c j}{2} \int_{0}^{\xi} e^{j q(\xi-\tau)} \int_{0}^{\tau} G(\tau, s) d s d \tau .
$$


Substitute the above result into (2.8), to obtain an integral equation for $G(\xi, \eta)$ :

$$
\begin{aligned}
G(\xi, \eta)= & -j q e^{j q \eta}-\frac{c j}{2} \int_{0}^{\eta} e^{j q(\eta-\tau)} d \tau+\frac{c j}{2} \int_{0}^{\eta} e^{j q(\eta-\tau)} \int_{0}^{\tau} G(\tau, s) d s d \tau \\
& -\frac{c j}{4}(\xi-\eta)+\frac{c j}{4} \int_{\eta}^{\xi} \int_{0}^{\eta} G(\tau, s) d s d \tau
\end{aligned}
$$

We now use the method of successive approximations to show that this equation has a unique continuous solution. Set

$$
\left\{\begin{array}{l}
G_{0}(\xi, \eta)=-j q e^{j q \eta}-\frac{c j}{2} \int_{0}^{\eta} e^{j q(\eta-\tau)} d \tau-\frac{c j}{4}(\xi-\eta) \\
G_{n}(\xi, \eta)=\frac{c j}{2} \int_{0}^{\eta} e^{j q(\eta-\tau)} \int_{0}^{\tau} G_{n-1}(\tau, s) d s d \tau+\frac{c j}{4} \int_{\eta}^{\xi} \int_{0}^{\eta} G_{n-1}(\tau, s) d s d \tau, n \geqslant 1 .
\end{array}\right.
$$

Then one can readily show that

$$
\left|G_{0}(\xi, \eta)\right| \leqslant q+\frac{c}{2} \eta+\frac{c}{4}|\xi-\eta| \leqslant 2 c+q=M .
$$

Suppose that

$$
\left|G_{n}(\xi, \eta)\right| \leqslant M^{n+1} \frac{(\xi+\eta)^{n}}{n !} .
$$

Then, we have the following estimate

$$
\begin{aligned}
\left|G_{n+1}\right| & \leqslant \frac{c M^{n+1}}{4 n !}\left|2 \int_{0}^{\eta} \int_{0}^{\tau}(\tau+s)^{n} d s d \tau+\int_{\eta}^{\xi} \int_{0}^{\eta}(\tau+s)^{n} d s d \tau\right| \\
& \leqslant \frac{c M^{n+1}}{4 n !} \frac{(\xi+\eta)^{n+1}}{n+1} \\
& \leqslant M^{n+2} \frac{(\xi+\eta)^{n+1}}{(n+1) !}
\end{aligned}
$$

By mathematical induction, (2.14) is true for all $n \geqslant 0$. It then follows from the Weierstrass M-test that the series

$$
G(\xi, \eta)=\sum_{n=0}^{\infty} G_{n}(\xi, \eta)
$$

converges absolutely and uniformly in $0 \leqslant \eta \leqslant \xi \leqslant 2$. Furthermore, by [19, Theorem 4.17, p.156], we deduce that

$$
\begin{aligned}
G(\xi, \eta) & =\sum_{n=0}^{\infty} G_{n}(\xi, \eta)=-j q e^{j q \eta}-\frac{c j}{2} \int_{0}^{\eta} e^{j q(\eta-\tau)} d \tau-\frac{c j}{4}(\xi-\eta) \\
& +\sum_{n=1}^{\infty} \frac{c j}{2} \int_{0}^{\eta} e^{j q(\eta-\tau)} \int_{0}^{\tau} G_{n-1}(\tau, s) d s d \tau+\sum_{n=1}^{\infty} \frac{c j}{4} \int_{\eta}^{\xi} \int_{0}^{\eta} G_{n-1}(\tau, s) d s d \tau \\
& =-j q e^{j q \eta}-\frac{c j}{2} \int_{0}^{\eta} e^{j q(\eta-\tau)} d \tau-\frac{c j}{4}(\xi-\eta) \\
& +\frac{c j}{2} \int_{0}^{\eta} e^{j q(\eta-\tau)} \int_{0}^{\tau} \sum_{n=1}^{\infty} G_{n-1}(\tau, s) d s d \tau+\frac{c j}{4} \int_{\eta}^{\xi} \int_{0}^{\eta} \sum_{n=1}^{\infty} G_{n-1}(\tau, s) d s d \tau \\
& =-j q e^{j q \eta}-\frac{c j}{2} \int_{0}^{\eta} e^{j q(\eta-\tau)} d \tau-\frac{c j}{4}(\xi-\eta) \\
& +\frac{c j}{2} \int_{0}^{\eta} e^{j q(\eta-\tau)} \int_{0}^{\tau} G(\tau, s) d s d \tau+\frac{c j}{4} \int_{\xi}^{\eta} \int_{0}^{\eta} G(\tau, s) d s d \tau .
\end{aligned}
$$


This shows that $G(\xi, \eta)$ is a continuous solution of Equation (2.12), and hence is twice continuously differentiable in $0 \leqslant \eta \leqslant \xi \leqslant 2$, and $|G(\xi, \eta)| \leqslant M \exp (M(\xi+\eta))$. We claim that this solution is unique. To this purpose, it suffices to show that the equation

$$
G(\xi, \eta)=\frac{c j}{2} \int_{0}^{\eta} e^{j q(\eta-\tau)} \int_{0}^{\tau} G(\tau, s) d s d \tau+\frac{c j}{4} \int_{\eta}^{\xi} \int_{0}^{\eta} G(\tau, s) d s d \tau
$$

has zero solution only. Define the mapping $F_{0}: \Omega_{0} \rightarrow \Omega_{0}, \Omega_{0}=\{G \mid G(\xi, \eta)$ is continuous in $0 \leqslant$ $\eta \leqslant \xi \leqslant 2\}:$

$$
\left(F_{0} G\right)(\xi, \eta)=\frac{c j}{2} \int_{0}^{\eta} e^{j q(\eta-\tau)} \int_{0}^{\tau} G(\tau, s) d s d \tau+\frac{c j}{4} \int_{\eta}^{\xi} \int_{0}^{\eta} G(\tau, s) d s d \tau, \forall G \in \Omega_{0} .
$$

Then $F_{0}$ is a compact operator on $\Omega_{0}$. By $(2.14)$, the spectral radius of $F_{0}$ is zero. So 0 is the unique spectrum of $F_{0}$. Therefore, (2.16) has zero solution only. The proof is complete.

Remark 2.1 The proof of Lemma 2.1 shows that

$$
\lim _{N \rightarrow \infty} \sum_{n=0}^{N} G_{n}(\xi, \eta)=G(\xi, \eta)=k(x, y), \xi=x+y, \eta=x-y
$$

uniformly in $x \in[0,1], y \in[0, x]$, where $G_{n}(\xi, \eta)$ is given by (2.13). This can be used to approximate the kernel function $k$ numerically.

The transformation (2.1) transforms system (1.1) into the following system:

$$
\left\{\begin{array}{l}
w_{t}(x, t)=-j w_{x x}(x, t)-c w(x, t), x \in(0,1), t>0, \\
w_{x}(0, t)=0, t \geqslant 0, \\
w_{x}(1, t)=U(t)+d(t)-k(1,1) u(1, t)-\int_{0}^{1} k_{x}(1, y) u(y, t) d y, t \geqslant 0 .
\end{array}\right.
$$

Set the sliding mode surface as

$$
S^{W}=\left\{f \in \mathcal{H} \mid \int_{0}^{1} f(x) d x=0\right\},
$$

which is a closed subspace of the state space $\mathcal{H}$. The corresponding sliding mode function for system $(2.18)$ is

$$
S_{W}(t)=\int_{0}^{1} w(x, t) d x
$$

On the sliding mode surface $S_{W}(t) \equiv 0$, the system (2.18) becomes

$$
\left\{\begin{array}{l}
w_{t}(x, t)=-j w_{x x}(x, t)-c w(x, t), x \in(0,1), t>0, \\
w_{x}(0, t)=\int_{0}^{1} w(x, t) d x=0, t \geqslant 0 .
\end{array}\right.
$$

The Proposition 2.1 below shows that system (2.21) decays exponentially in $\mathcal{H}$ as $t \rightarrow \infty$ with the decay rate $-c$. This is the advantage of the transformation (2.1). If we use the analytic backstepping transformation presented in exercise 6.2 of [17] on pages 76-77 (see (3.1) in next section), it is hard to find the sliding mode surface in $\mathcal{H}$. 
Proposition 2.1 The system (2.21) associates with a $C_{0}$-semigroup of contractions on $S^{W}$, and is exponentially stable in $S^{W}$ with the decay rate $-c$.

Proof. Define the operator $A: D(A)\left(\subset S^{W}\right) \rightarrow S^{W}$ as follows:

$$
\left\{\begin{array}{l}
A f(x)=-j f^{\prime \prime}(x)-c f(x), \forall f \in D(A), \\
D(A)=\left\{f \in S^{W} \cap H^{2}(0,1) \mid f^{\prime \prime} \in S^{W}, f^{\prime}(0)=0\right\} .
\end{array}\right.
$$

It is easy to show that for any $f \in D(A), f^{\prime \prime} \in S^{W}$ if and only if $f^{\prime}(1)=0$. So we can write $A$ as

$$
\left\{\begin{array}{l}
A f(x)=-j f^{\prime \prime}(x)-c f(x), \forall f \in D(A), \\
D(A)=\left\{f \in S^{W} \cap H^{2}(0,1) \mid f^{\prime}(0)=f^{\prime}(1)=0\right\} .
\end{array}\right.
$$

Now for any $f \in D(A)$,

$$
\operatorname{Re}\langle A f, f\rangle=-\operatorname{Re} j \int_{0}^{1} f^{\prime \prime}(x) \overline{f(x)} d x-c \int_{0}^{1}|f(x)|^{2} d x=-c \int_{0}^{1}|f(x)|^{2} d x \leqslant 0 .
$$

Hence $A+c I$ is dissipative and so is for $A$. For any $g \in S^{W}$ solve $A f=g$, that is

$$
\left\{\begin{array}{l}
-j f^{\prime \prime}(x)-c f(x)=g(x), \\
f^{\prime}(0)=f^{\prime}(1)=0
\end{array}\right.
$$

to get the unique solution $f$ as

$$
\left\{\begin{array}{l}
f(x)=c_{0}\left(e^{\frac{\sqrt{c}(j+1) x}{\sqrt{2}}}+e^{-\frac{\sqrt{c}(j+1) x}{\sqrt{2}}}\right)+\frac{1}{\sqrt{2 c}(j+1)} \int_{0}^{x}\left(e^{\frac{\sqrt{c}(j+1)}{\sqrt{2}}(x-s)}-e^{-\frac{\sqrt{c}(j+1)}{\sqrt{2}}(x-s)}\right) g(s) d s \\
c_{0}=-\frac{1}{\sqrt{2 c}(j+1)\left(e^{\frac{\sqrt{c}(j+1)}{\sqrt{2}}}+e^{-\frac{\sqrt{c}(j+1)}{\sqrt{2}}}\right)} \int_{0}^{1}\left(e^{\frac{\sqrt{c}(j+1)}{\sqrt{2}}(1-s)}-e^{-\frac{\sqrt{c}(j+1)}{\sqrt{2}}(1-s)}\right) d s
\end{array}\right.
$$

So, $A^{-1}$ exists and is bounded on $S^{W}$. By the Lumer-Phillips theorem ([24, Theorem 4.3,p.14]), $A$ generates a $C_{0}$-semigroup of contractions on $S^{W}$, and so does for $A+c I$. Therefore, the semigroup generated by $A$ is exponentially stable with the decay rate $-c$.

Now we are in a position to seek the finite time "reaching condition" for target system (2.18). Formally, it has

$$
\begin{aligned}
\dot{S}_{W}(t) & =\int_{0}^{1} w_{t}(x, t) d x=\int_{0}^{1}\left[-j w_{x x}(x, t)-c w(x, t)\right] d x \\
& =-j w_{x}(1, t)-c \int_{0}^{1} w(x, t) d x=-j w_{x}(1, t)-c S_{W}(t),
\end{aligned}
$$

and hence

$$
\dot{S}_{W}(t)=-j\left[U(t)+d(t)-k(1,1) u(1, t)-\int_{0}^{1} k_{x}(1, y) u(y, t) d y\right]-c S_{W}(t)
$$

Design the feedback controller:

$$
U(t)=k(1,1) u(1, t)+\int_{0}^{1} k_{x}(1, y) u(y, t) d y+c j S_{W}(t)+U_{0}(t),
$$


where $U_{0}$ is a new control. Then

$$
\dot{S}_{W}(t)=-j U_{0}(t)-j d(t)
$$

Let

$$
U_{0}(t)=-j\left(M_{0}+\eta\right) \frac{S_{W}(t)}{\left|S_{W}(t)\right|} \text { for } S_{W}(t) \neq 0
$$

Then

$$
\dot{S}_{W}(t)=-\left(M_{0}+\eta\right) \frac{S_{W}(t)}{\left|S_{W}(t)\right|}-j d(t) \text { for } S_{W}(t) \neq 0
$$

Therefore,

$$
\frac{d}{d t}\left|S_{W}(t)\right|^{2}=2 \operatorname{Re} \overline{S_{W}(t)} \dot{S}_{W}(t)=-2\left(M_{0}+\eta\right)\left|S_{W}(t)\right|-2 \operatorname{Re}\left(j d(t) S_{W}(t)\right) \leqslant-2 \eta\left|S_{W}(t)\right|,
$$

which is just the finite time "reaching condition" to be proved rigorously later. The sliding mode controller is

$$
U(t)=k(1,1) u(1, t)+\int_{0}^{1} k_{x}(1, y) u(y, t) d y+c j S_{W}(t)-j\left(M_{0}+\eta\right) \frac{S_{W}(t)}{\left|S_{W}(t)\right|} \text { for } S_{W}(t) \neq 0 .
$$

Under the control (2.27), the closed-loop of the target system (2.18) becomes

$$
\left\{\begin{array}{l}
w_{t}(x, t)=-j w_{x x}(x, t)-c w(x, t) \\
w_{x}(0, t)=0 \\
w_{x}(1, t)=c j S_{W}(t)-j\left(M_{0}+\eta\right) \frac{S_{W}(t)}{\left|S_{W}(t)\right|}+d(t)=c j S_{W}(t)+\tilde{d}(t), S_{W}(t) \neq 0,
\end{array}\right.
$$

where

$$
\tilde{d}(t)=-j\left(M_{0}+\eta\right) \frac{S_{W}(t)}{\left|S_{W}(t)\right|}+d(t) .
$$

The next result confirms the existence and uniqueness of the solution to (2.28) and the finite time "reaching condition" to the sliding mode surface $S^{W}$.

Proposition 2.2 Suppose that $d$ is measurable and $|d(t)| \leqslant M_{0}$ for all $t \geqslant 0$, and let $S_{W}$ be defined by (2.20). Then for any $w(\cdot, 0) \in \mathcal{H}, S_{W}(0) \neq 0$, there exists a $t_{\text {max }}>0$ such that (2.28) admits a unique solution $w \in C\left(0, t_{\max } ; \mathcal{H}\right)$ and $S_{W}(t)=0$ for all $t \geqslant t_{\max }$.

Proof. Write system (2.28) as

$$
\frac{d}{d t} w(\cdot, t)=\mathcal{A}_{0} w(\cdot, t)+\mathcal{B}_{0} \tilde{d}(t), \mathcal{B}_{0}=-j \delta(x-1),
$$

where $\mathcal{A}_{0}$ is given by

$$
\left\{\begin{array}{l}
\mathcal{A}_{0} f=-j f^{\prime \prime}-c f \\
D\left(\mathcal{A}_{0}\right)=\left\{f \in H^{2}(0,1) \mid f^{\prime}(0)=0, f^{\prime}(1)=c j \int_{0}^{1} f(x) d x\right\} .
\end{array}\right.
$$

We claim that $\mathcal{A}_{0}$ generates a $C_{0}$-semigroup on $\mathcal{H}$. To this purpose, it suffices to show that $\mathcal{A}_{0}^{*}$, the adjoint operator of $\mathcal{A}_{0}$, generates a $C_{0}$-semigroup on $\mathcal{H}$. 
A straightforward calculation shows that

$$
\left\{\begin{array}{l}
\mathcal{A}_{0}^{*} g=j g^{\prime \prime}-c g+c g(1), \\
D\left(\mathcal{A}_{0}^{*}\right)=\left\{g \in H^{2}(0,1) \mid g^{\prime}(0)=g^{\prime}(1)=0\right\} .
\end{array}\right.
$$

The dual system of (2.30) is hence given by

$$
\left\{\begin{array}{l}
w_{t}^{*}(x, t)=j w_{x x}^{*}(x, t)-c w^{*}(x, t)+c w^{*}(1, t), \\
w_{x}^{*}(0, t)=w_{x}^{*}(1, t)=0 \\
y(t)=\mathcal{B}_{0}^{*} w^{*}=-j w^{*}(1, t) .
\end{array}\right.
$$

It is an exercise to get the eigen-pairs $\left(\lambda_{n}, \varphi_{n}\right)$ of $\mathcal{A}_{0}^{*}$ are

$$
\lambda_{n}=-c-j(n \pi)^{2}, \varphi_{n}(x)=\cos n \pi x-c \frac{\cos n \pi}{c-j(n \pi)^{2}}, n=0,1,2, \cdots .
$$

By Bari's theorem, $\left\{\varphi_{n}\right\}_{n=0}^{\infty}$ forms a Riesz basis for $\mathcal{H}$. So $\mathcal{A}_{0}^{*}$ generates a $C_{0}$-semigroup on $\mathcal{H}$, and so does for $\mathcal{A}_{0}$. Moreover, for any $w^{*}(\cdot, 0) \in \mathcal{H}$, suppose that

$$
w^{*}(x, 0)=\sum_{n=0}^{\infty} a_{n} \varphi_{n}(x) .
$$

Then

$$
w^{*}(x, t)=e^{\mathcal{A}_{0}^{*} t} w^{*}(x, 0)=\sum_{n=0}^{\infty} e^{\lambda_{n} t} a_{n} \varphi_{n}(x),
$$

and hence

$$
y(t)=-j \sum_{n=0}^{\infty} e^{\lambda_{n} t} a_{n} \varphi_{n}(1) .
$$

By Ingham's inequality ([18, theorems 4.3]), there exists a $T>0$, such that

$$
\int_{0}^{T}|y(t)|^{2} d t \leqslant C_{T} \sum_{n=0}^{\infty}\left|a_{n} \varphi_{n}(1)\right|^{2} \leqslant D_{T}\left\|w^{*}(\cdot, 0)\right\|^{2}
$$

for some constants $C_{T}, D_{T}$ that depend on $T$ only. This shows that $\mathcal{B}_{0}^{*}$ is admissible for $e^{\mathcal{A}_{0}^{*} t}$ and so is $\mathcal{B}_{0}$ for $e^{\mathcal{A}_{0} t}([29,30])$. Therefore, for any $T>0$ and $w(\cdot, 0) \in \mathcal{H}$, if $S_{W} \in C[0, T], S_{W}(t) \neq 0$ for all $t \in[0, T)$, then there exists a unique solution $w \in C(0, T ; \mathcal{H})$ to $(2.28)$.

Suppose that $S_{W} \in C[0, T], S_{W}(t) \neq 0$ for all $t \in[0, T)$. Since $f(x) \equiv 1 \in D\left(A_{0}^{*}\right)$, by the admissibility just verified, take the inner product with $f=1$ on both sides of (2.30) to get

$$
\frac{d}{d t} \int_{0}^{1} w(x, t) d x=\left\langle w, \mathcal{A}_{0}^{*} f\right\rangle+\tilde{d}(t)\left\langle f, \mathcal{B}_{0}^{*} f\right\rangle=-j \tilde{d}(t)=-\left(M_{0}+\eta\right) \frac{S_{W}(t)}{\left|S_{W}(t)\right|}-j d(t), \forall t \in[0, T] \text { a.e. }
$$

or

$$
\dot{S}_{W}(t)=-\left(M_{0}+\eta\right) \frac{S_{W}(t)}{\left|S_{W}(t)\right|}-j d(t), \forall t \in[0, T] \text { a.e., }
$$

which is just (2.25). So if (2.35) admits a unique continuous, nonzero solution, then (2.28) admits a unique solution $w \in C(0, T ; \mathcal{H})$. 
Suppose that $t_{0} \geqslant 0$ and $S_{W}\left(t_{0}\right)=S_{0} \neq 0$. Then it follows from (2.35) that

$$
S_{W}(t)=S_{0}-\left(M_{0}+\eta\right) \int_{t_{0}}^{t} \frac{S_{W}(\tau)}{\left|S_{W}(\tau)\right|} d \tau-j \int_{t_{0}}^{t} d(\tau) d \tau, \forall t \geqslant t_{0} .
$$

Define a closed subspace of $C\left[t_{0}, t_{0}+\frac{\left|S_{0}\right|}{4\left(2 M_{0}+\eta\right)}\right]$ by

$$
\begin{aligned}
& \Omega=\left\{S \in C\left[t_{0}, t_{0}+\frac{\left|S_{0}\right|}{4\left(2 M_{0}+\eta\right)}\right] \mid\right. S\left(t_{0}\right)=S_{0}, \\
&\left.|S(t)| \geqslant \frac{3\left|S_{0}\right|}{4}, \forall t \in\left[t_{0}, t_{0}+\frac{\left|S_{0}\right|}{4\left(2 M_{0}+\eta\right)}\right]\right\},
\end{aligned}
$$

and a mapping $F$ on $\Omega$ by

$$
(F S)(t)=S_{0}-\left(M_{0}+\eta\right) \int_{t_{0}}^{t} \frac{S(\tau)}{|S(\tau)|} d \tau-j \int_{t_{0}}^{t} d(\tau) d \tau .
$$

Then for any $S \in \Omega$, it has

$$
|(F S)(t)| \geqslant\left|S_{0}\right|-\left(t-t_{0}\right)\left(2 M_{0}+\eta\right) \geqslant \frac{3\left|S_{0}\right|}{4} .
$$

This shows that $F \Omega \subset \Omega$. Moreover,

$$
\begin{aligned}
\left|\left(F S_{1}\right)(t)-\left(F S_{2}\right)(t)\right| & \leqslant\left(M_{0}+\eta\right) \int_{t_{0}}^{t}\left|\frac{S_{1}(\tau)}{\left|S_{1}(\tau)\right|}-\frac{S_{2}(\tau)}{\left|S_{2}(\tau)\right|}\right| d \tau \\
& \leqslant 2\left(M_{0}+\eta\right) \int_{t_{0}}^{t} \frac{\left|S_{1}(\tau)-S_{2}(\tau)\right|}{\left|S_{1}(\tau)\right|} \mid d \tau \leqslant \frac{2\left(M_{0}+\eta\right)}{3\left(2 M_{0}+\eta\right)}\left\|S_{1}-S_{2}\right\|_{\Omega}
\end{aligned}
$$

where $\|S\|_{\Omega}=\|S\|_{C\left[t_{0}, t_{0}+\frac{\left|S_{0}\right|}{4\left(2 M_{0}+\eta\right)}\right]}$. The aforementioned inequality shows that the mapping $F$ defined by (2.38) is a contraction mapping on $\Omega$. By the contraction mapping principle, there exists a unique, nonzero solution $S_{W}$ to $(2.36)$ in $C\left[t_{0}, t_{0}+\frac{\left|S_{0}\right|}{4\left(2 M_{0}+\eta\right)}\right]$.

The aforementioned arguments show that when $S_{W}(0) \neq 0$, there exists a unique continuous solution $S_{W}$ to $(2.35)$ in the maximal interval $\left[0, t_{\max }\right)$, where it must have $S_{W}\left(t_{\max }\right)=0$. It then follows from (2.26) that $\left|S_{W}(t)\right|$ must be decreasing in $\left[0, t_{\max }\right)$ and $\left|S_{W}(t)\right|>0$ for all $t \in\left[0, t_{\max }\right)$. Since $S_{W}(t)$ is continuous, the reaching condition $(2.26)$ implies that $S_{W}(t) \equiv 0$ for all $t \geqslant t_{\max }$. The proof is complete.

Returning back to the system (1.1) under the transformation (2.1), feedback control (2.27), we obtain the main result of this section from Proposition 2.2.

Theorem 2.1 Suppose that $d$ is measurable and $|d(t)| \leqslant M_{0}$ for all $t \geqslant 0$, and let $S_{U}$ be the sliding mode function given by

$$
S_{U}(t)=\int_{0}^{1} u(x, t) d x-\int_{0}^{1} \int_{0}^{x} k(x, y) u(y, t) d y d x .
$$


Then for any $u(\cdot, 0) \in \mathcal{H}, S_{U}(0) \neq 0$, there exists a $t_{\text {max }}>0$ such that the closed-loop system of (1.1) under the feedback control (2.27) is

$$
\left\{\begin{aligned}
u_{t}(x, t) & =-j u_{x x}(x, t) \\
u_{x}(0, t) & =-j q u(0, t) \\
u_{x}(1, t) & =k(1,1) u(1, t)+\int_{0}^{1} k_{x}(1, y) u(y, t) d y+c j S_{U}(t) \\
& -j\left(M_{0}+\eta\right) \frac{S_{U}(t)}{\left|S_{U}(t)\right|}+d(t), S_{U}(t) \neq 0
\end{aligned}\right.
$$

which admits a unique solution $u \in C\left(0, t_{\max } ; \mathcal{H}\right)$ and $S_{U}(t)=0$ for all $t \geqslant t_{\text {max }}$. On the sliding mode surface $S_{U}(t)=0$, the system (1.1) becomes

$$
\left\{\begin{array}{l}
u_{t}(x, t)=-j u_{x x}(x, t) \\
u_{x}(0, t)=-j q u(0, t) \\
\int_{0}^{1} u(x, t) d x-\int_{0}^{1} \int_{0}^{x} k(x, y) u(y, t) d y d x=0
\end{array}\right.
$$

which is equivalent to (2.21) and hence is exponentially stable in $\mathcal{H}$ with the decay rate $-c$.

It is remarked that system (2.28) is equivalent to system (2.40) under the equivalent transformation (2.1).

\section{The active disturbance rejection control approach}

In this section, we suppose in addition that $|\dot{d}|$ is also uniformly bounded. Following exercise 6.2 of [17] on pages $76-77$, we introduce a transformation:

$$
w(x, t)=u(x, t)+j\left(c_{0}+q\right) \int_{0}^{x} e^{j q(x-y)} u(y, t) d y, c_{0}>0 .
$$

Its inverse transformation is found to be

$$
u(x, t)=w(x, t)-j\left(c_{0}+q\right) \int_{0}^{x} e^{-j c_{0}(x-y)} w(y, t) d y .
$$

The transformation (3.1) transforms system (1.1) into the following system:

$$
\left\{\begin{array}{l}
w_{t}(x, t)=-j w_{x x}(x, t), x \in(0,1), t>0 \\
w_{x}(0, t)=j c_{0} w(0, t), t \geqslant 0 \\
w_{x}(1, t)=U(t)+d(t)+j\left(c_{0}+q\right) w(1, t)+c_{0}\left(c_{0}+q\right) \int_{0}^{1} e^{-j c_{0}(1-x)} w(x, t) d x, t \geqslant 0 .
\end{array}\right.
$$

It is seen that the anti-stable factor $-j q u(0, t)$ in (1.1) becomes the dissipative term $j c_{0} w(0, t)$ in (3.3) under the transformation (3.1), both at the end $x=0$. In what follows, we consider 
the stabilization of system (3.3) until the final step to go back the system (1.1) under the inverse transformation (3.2). Introduce a new controller $U_{0}(t)$ so that

$$
U(t)=U_{0}(t)-j\left(c_{0}+q\right) w(1, t)-c_{0}\left(c_{0}+q\right) \int_{0}^{1} e^{-j c_{0}(1-x)} w(x, t) d x .
$$

Then (3.3) becomes

$$
\left\{\begin{array}{l}
w_{t}(x, t)=-j w_{x x}(x, t), x \in(0,1), t>0, \\
w_{x}(0, t)=j c_{0} w(0, t), t \geqslant 0, \\
w_{x}(1, t)=U_{0}(t)+d(t) .
\end{array}\right.
$$

We write (3.5) into the operator form. Define the operator $\mathcal{A}$ as follows:

$$
\left\{\begin{array}{l}
\mathcal{A} f(x)=-j f^{\prime \prime}(x), \\
D(\mathcal{A})=\left\{f \in H^{2}(0,1) \mid f^{\prime}(0)=j c_{0} f(0), f^{\prime}(1)=0\right\} .
\end{array}\right.
$$

Its adjoint $\mathcal{A}^{*}$ is found to be

$$
\left\{\begin{array}{l}
\mathcal{A}^{*} f(x)=j f^{\prime \prime}(x), \\
D\left(\mathcal{A}^{*}\right)=\left\{f \in H^{2}(0,1) \mid f^{\prime}(0)=-j c_{0} f(0), f^{\prime}(1)=0\right\},
\end{array}\right.
$$

with

$$
\mathcal{A}^{*-1} f=C-j \int_{1}^{x}(x-\tau) f(\tau) d \tau, C=-\frac{1}{c_{0}} \int_{0}^{1} f(x) d x+j \int_{0}^{1} x f(x) d x, \forall f \in \mathcal{H} .
$$

Then we can write $(3.5)$ in $\mathcal{H}$ as

$$
\frac{d}{d t} w(\cdot, t)=\mathcal{A} w(\cdot, t)+\mathcal{B}\left(U_{0}(t)+d(t)\right), \mathcal{B}=-j \delta(x-1) .
$$

Lemma 3.1 Let $\mathcal{A}$ be defined by (3.6). Then each eigenvalue of $\mathcal{A}$ is algebraically simple, and there exists a sequence of eigenfunctions of $\mathcal{A}$, which form a Riesz basis for $\mathcal{H}$. Therefore, $\mathcal{A}$ generates an exponential stable $C_{0}$-semigroup on $\mathcal{H}$.

Proof. Solve $\mathcal{A} f=-j \lambda^{2} f$, that is,

$$
\left\{\begin{array}{l}
f^{\prime \prime}(x)=\lambda^{2} f(x) \\
f^{\prime}(0)=j c_{0} f(0), f^{\prime}(1)=0
\end{array}\right.
$$

to get

$$
f(x)=\frac{\lambda+j c_{0}}{\lambda-j c_{0}} e^{\lambda x}+e^{-\lambda x}
$$

where $\lambda$ satisfies

$$
e^{2 \lambda}=\frac{\lambda-j c_{0}}{\lambda+j c_{0}}=1-\frac{2 j c_{0}}{\lambda}+\mathcal{O}\left(|\lambda|^{-2}\right) \text { as }|\lambda| \rightarrow \infty .
$$

Notice that $\lambda \neq \pm j c_{0}, \lambda \neq 0$ for nonzero $f$ by (3.10). Solve $\left(-j \lambda^{2}-A\right) \varphi=f$ where $f$ is given by (3.11), that is

$$
\left\{\begin{array}{l}
\varphi^{\prime \prime}(x)=\lambda^{2} \varphi(x)-j f(x), \\
\varphi^{\prime}(0)=j c_{0} \varphi(0), \varphi^{\prime}(1)=0,
\end{array}\right.
$$


to get

$$
\varphi(x)=\tilde{c}\left[\frac{\lambda+j c_{0}}{\lambda-j c_{0}} e^{\lambda x}+e^{-\lambda x}\right]-j \int_{0}^{x} \frac{e^{\lambda(x-s)}-e^{-\lambda(x-s)}}{2 \lambda} f(s) d s
$$

for general constant $\tilde{c}$. By $\varphi^{\prime}(1)=0$, it follows that

$$
\int_{0}^{1}\left[e^{\lambda(1-s)}+e^{-\lambda(1-s)}\right] f(s) d s=0 .
$$

Substitute (3.11) into above to get

$$
2 e^{-\lambda} \frac{\lambda^{2}-j c_{0}+c_{0}^{2}}{\lambda^{2}+c_{0}^{2}}=\frac{e^{-\lambda}}{2 \lambda}\left(4 \lambda+e^{2 \lambda}-e^{-2 \lambda}\right)=0 .
$$

So $\lambda^{2}=j c_{0}-c_{0}^{2}$ and $e^{2 \lambda}=-2 \lambda \pm \sqrt{4 \lambda^{2}+1}$. But $\lambda= \pm \sqrt{j c_{0}-c_{0}^{2}}$ does not satisfy the characteristic equation (3.12) by Mathematica. Hence each eigenvalue of $\mathcal{A}$ is algebraically simple.

Next by the second equality of (3.12), it has

$$
\lambda=n \pi j+\mathcal{O}\left(n^{-1}\right), n \rightarrow \infty .
$$

Substitute (3.14) into (3.12) to obtain $\mathcal{O}\left(n^{-1}\right)=-c_{0} /(n \pi)$ and so

$$
\lambda=n \pi j-\frac{c_{0}}{n \pi}+\mathcal{O}\left(n^{-2}\right),-j \lambda^{2}=-2 c_{0}+j(n \pi)^{2}+\mathcal{O}\left(n^{-1}\right), n \rightarrow \infty .
$$

It is straightforward to verify that all eigenvalues of $\mathcal{A}$ have negative real parts. This also explains that why the system (1.1) is anti-stable: all eigenvalues of the free system (replace $c_{0}$ by $-q$ ) are located on the right half complex plane.

From (3.14) and (3.11), we get

$$
f(x)=\cos n \pi x+\mathcal{O}\left(n^{-1}\right), n \rightarrow \infty .
$$

Since from (3.8), $\mathcal{A}^{*-1}$ is compact on $\mathcal{H}$ and so is $\mathcal{A}^{-1}$. Hence $\mathcal{A}$ is a discrete operator. Since $\{\cos n \pi x\}_{n=0}^{\infty}$ forms an orthonormal basis for $\mathcal{H}$, it follows from theorem 6.3 of [12] and (3.16) that there is a sequence of the eigenfunctions of $\mathcal{A}$ which form a Riesz basis for $\mathcal{H}$. The proof is complete.

Lemma 3.2 Let $\mathcal{A}$ and $\mathcal{B}$ be defined in (3.9). Then $\mathcal{B}$ is admissible to the semigroup $e^{\mathcal{A} t}$.

Proof. By (3.8), we have

$$
\mathcal{B}^{*} \mathcal{A}^{*-1} f=-j C
$$

which is bounded from $\mathcal{H}$ to $\mathbb{C}$. Consider the dual system of (3.9):

$$
\left\{\begin{array}{l}
\frac{d}{d t} w^{*}(\cdot, t)=\mathcal{A}^{*} w^{*}(\cdot, t), \\
y(t)=B^{*} w^{*}(\cdot, t),
\end{array}\right.
$$


that is,

$$
\left\{\begin{array}{l}
w_{t}^{*}(x, t)=j w_{x x}^{*}(x, t), x \in(0,1), t>0 \\
w_{x}^{*}(0, t)=-j c_{0} w^{*}(0, t), t \geqslant 0 \\
w_{x}^{*}(1, t)=0, t \geqslant 0 \\
y(t)=-j w^{*}(1, t)
\end{array}\right.
$$

By (3.15) and (3.16), the eigen-pairs $\left\{\mu_{n}, g_{n}\right\}$ of $\mathcal{A}^{*}$ are

$$
\mu_{n}=j \bar{\lambda}^{2}=-2 c_{0}-j(n \pi)^{2}+\mathcal{O}\left(n^{-1}\right), g_{n}(x)=\cos n \pi x+\mathcal{O}\left(n^{-1}\right), n \rightarrow \infty .
$$

By Lemma 3.1, $\left\{g_{n}\right\}$ forms a Riesz basis for $\mathcal{H}$, so the solution $w$ of (3.17) can be written as

$$
w^{*}(x, t)=\sum_{n=0}^{\infty} b_{n} e^{\mu_{n} t} g_{n}(x) .
$$

Hence

$$
y(t)=-j \sum_{n=0}^{\infty} b_{n} e^{\mu_{n} t} g_{n}(1) .
$$

Same to (2.34), there exists a $T>0$ such that

$$
\int_{0}^{T}|y(t)|^{2} d t \leqslant C_{0 T} \sum_{n=0}^{\infty}\left|b_{n} g_{n}(1)\right|^{2} \leqslant C_{1 T} \sum_{n=0}^{\infty}\left|b_{n}\right|^{2} \leqslant C_{2 T}\left\|w^{*}(\cdot, 0)\right\|^{2}
$$

for some constants $C_{i T}, i=0,1,2$ that depend on $T$ only. This together with boundedness of $\mathcal{B}^{*} \mathcal{A}^{*-1}$ shows that $\mathcal{B}$ is admissible to the semigroup generated by $\mathcal{A}([29,30])$.

Let

$$
y_{1}(t)=\int_{0}^{1}\left(2 x^{3}-3 x^{2}\right) w(x, t) d x, \quad y_{2}(t)=\int_{0}^{1}(12 x-6) w(x, t) d x .
$$

Since $\mathcal{B}$ is admissible to the $C_{0}$-semigroup $e^{\mathcal{A} t}$, the solution of (3.5) is understood in the sense of

$$
\frac{d}{d t}\langle w(\cdot, t), f\rangle=\left\langle w(\cdot, t), \mathcal{A}^{*} f\right\rangle-j f(1)\left(U_{0}(t)+d(t)\right), \forall f \in D\left(\mathcal{A}^{*}\right) .
$$

Let $f(x)=2 x^{3}-3 x^{2} \in D\left(\mathcal{A}^{*}\right)$ in (3.19) to get

$$
\dot{y}_{1}(t)=j U_{0}(t)+j d(t)-j y_{2}(t) .
$$

That is to say, for any initial value $w(\cdot, 0) \in \mathcal{H}$, the (weak) solution of (3.5) must satisfy (3.20).

Remark 3.1 From (3.19), $y_{1}, y_{2}$ can be chosen as $y_{1}(t)=\int_{0}^{1} f(x) w(x, t) d x, y_{2}(t)=\int_{0}^{1}\left(\mathcal{A}^{*} f\right)(x) w(x, t) d x$ where $f \in D\left(\mathcal{A}^{*}\right), f(1) \neq 0$. Our choice is only a special case by this general principle.

Design the high gain estimators for $y_{1}$ and $d$ as follows:

$$
\left\{\begin{array}{l}
\dot{\hat{y}}(t)=j\left(U_{0}(t)+\hat{d}(t)\right)-j y_{2}(t)-\frac{1}{\varepsilon}\left(\hat{y}(t)-y_{1}(t)\right), \\
\dot{\hat{d}}(t)=\frac{j}{\varepsilon^{2}}\left(\hat{y}(t)-y_{1}(t)\right)
\end{array}\right.
$$


where $\varepsilon>0$ is the design small parameter and $\hat{d}$ is regarded as an approximation of $d$. Let

$$
\tilde{y}(t)=\hat{y}(t)-y_{1}(t), \tilde{d}(t)=j(\hat{d}(t)-d(t))
$$

be the errors. Then $\tilde{y}, \tilde{d}$ satisfy

$$
\frac{d}{d t}\left(\begin{array}{c}
\tilde{y}(t) \\
\tilde{d}(t)
\end{array}\right)=\left(\begin{array}{cc}
-\frac{1}{\varepsilon} & 1 \\
-\frac{1}{\varepsilon^{2}} & 0
\end{array}\right)\left(\begin{array}{c}
\tilde{y}(t) \\
\tilde{d}(t)
\end{array}\right)+\left(\begin{array}{c}
0 \\
-j
\end{array}\right) \dot{d}(t)=A\left(\begin{array}{c}
\tilde{y}(t) \\
\tilde{d}(t)
\end{array}\right)+B \dot{d}(t) .
$$

The eigenvalues of $A$ are found to be

$$
\lambda=-\frac{1}{2 \varepsilon} \pm \frac{\sqrt{3}}{2 \varepsilon} j .
$$

The state feedback controller to (3.5) is designed as follows:

$$
U_{0}(t)=-\hat{d}(t)
$$

It is clearly seen from (3.25) that this controller is just used to cancel the disturbance $d$ since $\mathcal{A}$ generates an exponential stable $C_{0}$-semigroup. This estimation/cancelation strategy (3.25) is obviously an economic strategy. Under the feedback (3.25), the closed-loop system of (3.5) becomes

$$
\left\{\begin{array}{l}
w_{t}(x, t)=-j w_{x x}(x, t), \quad x \in(0,1), \quad t>0, \\
w_{x}(0, t)=j c_{0} w(0, t), \quad t \geqslant 0, \\
w_{x}(1, t)=-\hat{d}(t)+d(t), \quad t \geqslant 0 . \\
\dot{\hat{y}}(t)=-j y_{2}(t)-\frac{1}{\varepsilon}\left(\hat{y}(t)-y_{1}(t)\right), \\
\dot{\hat{d}}(t)=\frac{j}{\varepsilon^{2}}\left(\hat{y}(t)-y_{1}(t)\right) .
\end{array}\right.
$$

Proposition 3.1 Suppose that $|d| \leqslant M_{0}$ and $\dot{d}$ is also uniformly bounded measurable. Then for any initial value $w(\cdot, 0) \in \mathcal{H}$, the closed-loop system (3.26) of (3.5) admits a unique solution $\left(w, w_{t}\right)^{\top} \in C(0, \infty ; \mathcal{H})$. Moreover, the solution of system (3.26) tends to any arbitrary given vicinity of zero as $t \rightarrow \infty, \varepsilon \rightarrow 0$.

Proof. Using the error variables $(\tilde{y}, \tilde{d})$ defined in $(3.22)$, we can write the equivalent system of (3.26) as follows:

$$
\left\{\begin{array}{l}
w_{t}(x, t)=-j w_{x x}(x, t), \quad x \in(0,1), \quad t>0, \\
w_{x}(0, t)=j c_{0} w(0, t), \quad t \geqslant 0 \\
w_{x}(1, t)=j \tilde{d}(t), \quad t \geqslant 0 . \\
\dot{\tilde{y}}(t)=-\frac{1}{\varepsilon} \tilde{y}(t)+\tilde{d}(t), t \geqslant 0 \\
\dot{\tilde{d}}(t)=-\frac{1}{\varepsilon^{2}} \tilde{y}(t)-j \dot{d}(t), t \geqslant 0 .
\end{array}\right.
$$


It is seen from $(3.27)$ that $(\tilde{y}, \tilde{d})$ is an external model for the " $w$ part" of the system $([22])$. So we can solve this ODE separately to be

$$
\left(\begin{array}{c}
\tilde{y}(t) \\
\tilde{d}(t)
\end{array}\right)=e^{A t}\left(\begin{array}{c}
\tilde{y}(0) \\
\tilde{d}(0)
\end{array}\right)+\int_{0}^{t} e^{A(t-s)} B \dot{d}(s) d s,
$$

where $A, B$ are defined in (3.23). By (3.24), a simple computation shows that the solution $(\tilde{y}, \tilde{d})$ of (3.28) satisfies

$$
(\tilde{y}(t), \tilde{d}(t)) \rightarrow 0 \text { as } t \rightarrow \infty, \varepsilon \rightarrow 0 .
$$

Now we consider the " $w$ part" of the system (3.27) which is re-written as

$$
\left\{\begin{array}{l}
w_{t}(x, t)=-j w_{x x}(x, t), x \in(0,1), t>0 \\
w_{x}(0, t)=j c_{0} w(0, t), t \geqslant 0 \\
w_{x}(1, t)=j \tilde{d}(t), t \geqslant 0
\end{array}\right.
$$

System (3.30) can be rewritten as an evolution equation in $\mathcal{H}$ as

$$
\frac{d}{d t} w(\cdot, t)=\mathcal{A} w(\cdot, t)+j \mathcal{B} \tilde{d}(t),
$$

where $\mathcal{A}, \mathcal{B}$ are the same as that in (3.9).

Since $\mathcal{A}$ generates an exponential stable $C_{0^{-}}$-semigroup on $\mathcal{H}$ (Lemma 3.1), and $\mathcal{B}$ is admissible to $e^{\mathcal{A} t}$ (Lemma 3.2), for any initial value $w(\cdot, 0) \in \mathcal{H}$, there exists a unique solution $w \in C(0, \infty ; \mathcal{H})$ which can be written as

$$
w(\cdot, t)=e^{\mathcal{A} t} w(\cdot, 0)+j \int_{0}^{t} e^{\mathcal{A}(t-s)} \mathcal{B} \tilde{d}(s) d s
$$

By (3.29), for any given $\varepsilon_{0}>0$, there exist $t_{0}>0$ and $\varepsilon_{1}>0$ such that $|\tilde{d}(t)|<\varepsilon_{0}$ for all $t>t_{0}$ and $0<\varepsilon<\varepsilon_{1}$. We rewrite solution of (3.32) as

$$
w(\cdot, t)=e^{\mathcal{A} t} w(\cdot, 0)+j e^{\mathcal{A}\left(t-t_{0}\right)} \int_{0}^{t_{0}} e^{\mathcal{A}\left(t_{0}-s\right)} \mathcal{B} \tilde{d}(s) d s+j \int_{t_{0}}^{t} e^{\mathcal{A}(t-s)} \mathcal{B} \tilde{d}(s) d s .
$$

The admissibility of $\mathcal{B}$ implies that

$$
\left\|\int_{0}^{t} e^{\mathcal{A}(t-s)} \mathcal{B} \tilde{d}(s) d s\right\|_{\mathcal{H}}^{2} \leqslant C_{t}\|\tilde{d}\|_{L_{l o c}^{2}(0, t)}^{2} \leqslant t^{2} C_{t}\|\tilde{d}\|_{L^{\infty}(0, t)}^{2}, \forall \tilde{d} \in L^{\infty}(0, \infty)
$$

for some constant $C_{t}$ that is independent of $\tilde{d}$. Since $e^{\mathcal{A} t}$ is exponentially stable, it follows from proposition 2.5 of [30] that

$$
\left\|\int_{t_{0}}^{t} e^{\mathcal{A}(t-s)} \mathcal{B} \tilde{d}(s) d s\right\|=\| \int_{0}^{t} e^{\mathcal{A}(t-s)} \mathcal{B}\left(0 \underset{t_{0}}{\diamond \tilde{d})(s) d s}\|\leqslant L\| \tilde{d} \|_{L^{\infty}(0, \infty)} \leqslant L \varepsilon_{0},\right.
$$

where $L$ is a constant that is independent of $\tilde{d}$, and

$$
\left(d_{1} \underset{\tau}{\diamond} d_{2}\right)(t)=\left\{\begin{array}{l}
d_{1}(t), 0 \leqslant t \leqslant \tau, \\
d_{2}(t-\tau), t>\tau,
\end{array}\right.
$$


where the left-hand side of (3.36) denotes the $\tau$-concatenation of $d_{1}$ and $d_{2}$ ([29]). Suppose that $\left\|e^{\mathcal{A} t}\right\| \leqslant L_{0} e^{-\omega t}$ for some $L_{0}, \omega>0$. By (3.33), (3.34), and (3.35), we have

$$
\|w(\cdot, t)\| \leqslant L_{0} e^{-\omega t}\|w(\cdot, 0)\|+L_{0} C_{t_{0}} e^{-\omega\left(t-t_{0}\right)}\|\tilde{d}\|_{L^{\infty}\left(0, t_{0}\right)}+L \varepsilon_{0} .
$$

As $t \rightarrow \infty$, the first two terms of (3.37) tend to zero. The result is then proved by the arbitrariness of $\varepsilon_{0}$.

Returning back to system (1.1) by the inverse transformation (3.2), feedback control (3.4) and (3.25), and new variable (3.18), we have proved, from Proposition 3.1, the main result of this section.

Theorem 3.1 Suppose that $|d| \leqslant M_{0}$ and $\dot{d}$ is also uniformly bounded measurable. Then for any initial value $u(\cdot, 0) \in \mathcal{H}$, the closed-loop system of (1.1) following:

$$
\left\{\begin{array}{l}
u_{t}(x, t)=-j u_{x x}(x, t), x \in(0,1), t>0, \\
u_{x}(0, t)=-j q u(0, t), t \geqslant 0 \\
u_{x}(1, t)=-\hat{d}(t)-j\left(c_{0}+q\right) u(1, t)+q\left(c_{0}+q\right) \int_{0}^{1} e^{j q(1-x)} u(x, t) d x+d(t), t \geqslant 0,
\end{array}\right.
$$

admits a unique solution $\left(u, u_{t}\right)^{\top} \in C(0, \infty ; \mathcal{H})$, and the solution of system (3.38) tends to any arbitrary given vicinity of zero as $t \rightarrow \infty, \varepsilon \rightarrow 0$, where the feedback control is:

$$
U(t)=-\hat{d}(t)-j\left(c_{0}+q\right) u(1, t)+q\left(c_{0}+q\right) \int_{0}^{1} e^{j q(1-x)} u(x, t) d x, t \geqslant 0
$$

and $\hat{d}$ satisfies

$$
\begin{gathered}
\left\{\begin{array}{l}
\dot{\hat{y}}(t)=-j y_{2}(t)-\frac{1}{\varepsilon}\left(\hat{y}(t)-y_{1}(t)\right) \\
\dot{\hat{d}}(t)=\frac{j}{\varepsilon^{2}}\left(\hat{y}(t)-y_{1}(t)\right)
\end{array}\right. \\
\left\{\begin{array}{l}
y_{1}(t)=\int_{0}^{1}\left(2 x^{3}-3 x^{2}\right)\left[u(x, t)+j\left(c_{0}+q\right) \int_{0}^{x} e^{j q(x-y)} u(y, t) d y\right] d x \\
y_{2}(t)=\int_{0}^{1}(12 x-6)\left[u(x, t)+j\left(c_{0}+q\right) \int_{0}^{x} e^{j q(x-y)} u(y, t) d y\right] d x .
\end{array}\right.
\end{gathered}
$$

\section{Numerical simulations}

In this section, the finite difference method is applied to compute the real and imaginary parts of the displacements numerically for both SMC and ADRC to illustrate the effect of the controllers. Figures 1(a) and 1(b) show the real and imaginary parts of displacement of system (2.40). Here the steps of space and time are taken as 0.1 and 0.0001 , respectively. We choose $q=1, c=10, M_{0}=4$, $\eta=1, u(x, 0)=10 x^{3}-2 j x^{2}$, and $d=2 \sin t$. The kernel function is approximated by (2.17) with $N=20$. Figures 2(a) and 2(b) show the real and imaginary parts of controller by SMC. Due to discontinuity, the the control vibrates rapidly after some time. 
Figures 3(a) and 3(b) plot the real and imaginary parts of system (3.38) with the same space and time sizes used in SMC. Other parameters are $q=c_{0}=1, \varepsilon=0.01, u(x, 0)=x+j x^{2}$, $d(t)=\cos t+j \sin t$. It is seen that in both cases, the displacements are obviously convergent. Moreover, Figures 4(a) and 4(b) show that convergence of $\hat{d}$ to the disturbance $d$. Figures 5(a) and 5(b) show the controller by ADRC. It is much better than that by SMC.

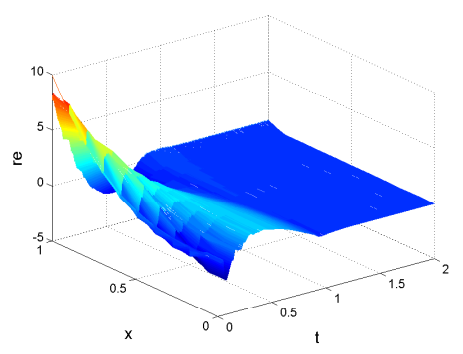

(a) Real part

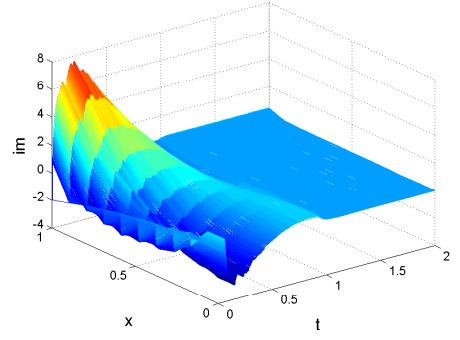

(b) Imaginary part

Figure 1: Real and imaginary parts of displacement with $d(t)=2 \sin t$ by SMC

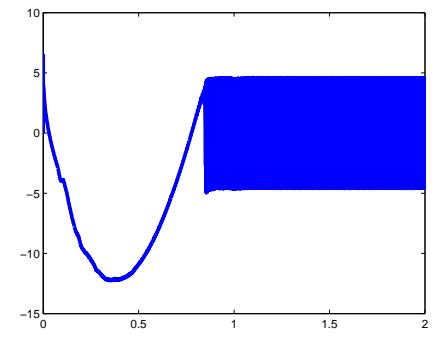

(a) Real part

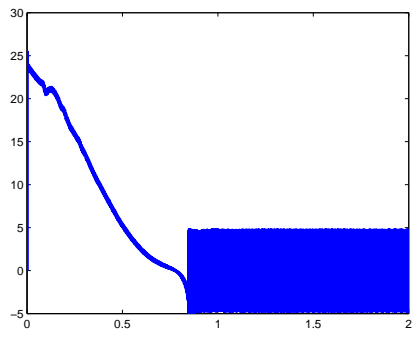

(b) Imaginary part

Figure 2: Real and imaginary parts of controller by SMC

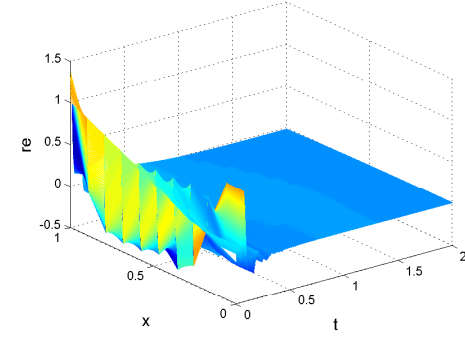

(a) Real part

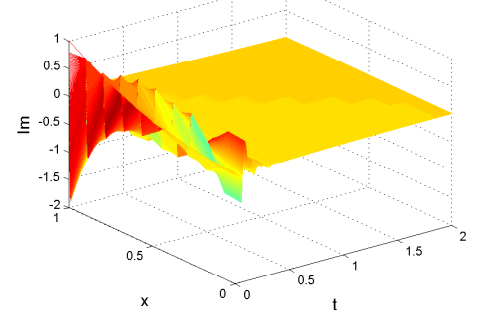

(b) Imaginary part

Figure 3: Real and imaginary parts of displacement with $d(t)=2 \sin t$ by ADRC 


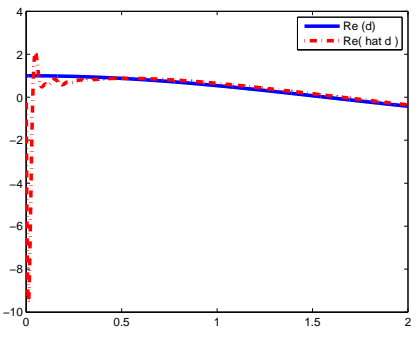

(a) Real part

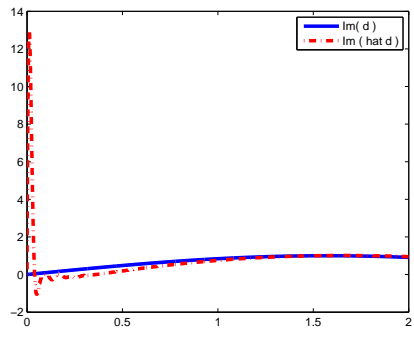

(b) Imaginary part

Figure 4: Real and imaginary parts of $\hat{d}(t)$ and disturbance $d(t)=\cos t+j \sin t$ by ADRC

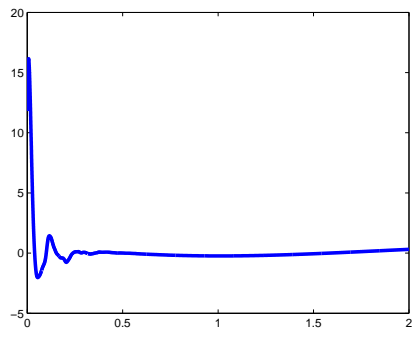

(a) Real part

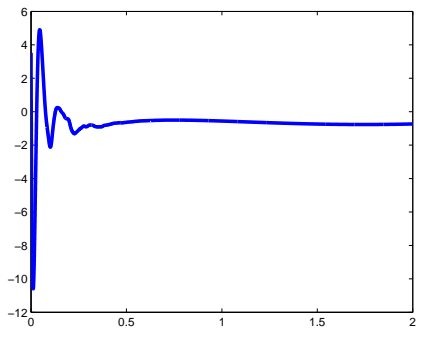

(b) Imaginary part

Figure 5: Real and imaginary parts of the controller by ADRC

\section{Concluding remarks}

In this paper, we apply two different approaches to stabilize a one-dimensional anti-stable Schrödinger equation subject to boundary control matched disturbance. We first apply the sliding mode control (SMC) approach to reject the disturbance. Since in SMC approach, we do not need to estimate the disturbance, the disturbance is assumed to be bounded only. The sliding mode surface is found to be a closed subspace of the state space. The closed-loop system is shown to have a unique (weak) solution and can reach the sliding mode surface in finite time. On the sliding mode surface, the system is shown to be exponentially stable with arbitrary prescribed decay rate. Owing to its complex valued nature, the "reaching condition" for Schrödinger equation is much more difficult than that for wave and beam equations.

The active disturbance rejection control (ADRC) is introduced to attenuate the disturbance. The disturbance is supposed additionally to have bounded derivative. The ADRC is an online estimation/cacellation control strategy, and we design a high gain estimator to estimate the disturbance. The smooth feedback control is designed (observed-based feedback control in some sense) which contains two parts. The first part is used to cancel the disturbance and the second part is to counter the anti-stability. The well-posedness of the closed-loop system is presented. It is shown that the closed-loop system can reach any arbitrary given vicinity of zero as time goes to infinity and high gain tuning parameter goes to zero. 


\section{References}

[1] A.M. Breger, A. G. Butkovskii, V. A. Kubyshkin, and V.I. Utkin, Sliding modes for control of distributed parameter entities subjected to a mobile multicycle signal, Automat. Remote Control, 41(1980), 346-355.

[2] Roger W. Brockett, New issues in the mathematics of control, in: "Mathematics Unlimited2001 and Beyond" (B. Engquist and W. Schmid, eds.), Springer-Verlag, Berlin, 2001, 189-219.

[3] G. Chen, M.C. Delfour, A.M. Krall, and G. Payre, Modeling, stabilization and control of serially connected beam, SIAM J.Control Optim., 25(1987), 526-546.

[4] M.B. Cheng, V. Radisavljevic and W.C. Su, Sliding mode boundary control of a parabolic PDE system with parameter variations and boundary uncertainties, Automatica, 47(2011), 381-387.

[5] S. Drakunov, E. Barbieeri and D.A. Silver, Sliding mode control of a heat equation with application to arc welding, IEEE International Conference on Control Applications, 1996, 668-672.

[6] B.Z. Guo and C.Z. Xu, The stabilization of a one-dimensional wave equation by boundary feedback with noncollocated observation, IEEE Trans. Automat. Control, 52(2007), 371-377.

[7] W. Guo, B.Z. Guo and Z.C. Shao, Parameter estimation and stabilization for a wave equation with boundary output harmonic disturbance and non-collocated control, Internat. J. Robust Nonlinear Control, 21(2011), 1297-1321.

[8] W. Guo and B.Z. Guo, Stabilization and regulator design for a one-dimensional unstable wave equation with input harmonic disturbance, Internat. J. Robust Nonlinear Control, in press.

[9] B.Z. Guo and F.F. Jin, Sliding mode and active disturbance rejection control to the stabilization of anti-stable one-dimensional wave equation subject to boundary input disturbance, IEEE Trans. Automat. Control, to appear.

[10] B.Z. Guo and Z.L. Zhao, On the convergence of extended state observer for nonlinear systems with uncertainty, Systems Control Lett., 60(2011), 420-430.

[11] B.Z. Guo and W. Kang, The Lyapunov approach to boundary stabilization of an anti-stable one-dimensional wave equation with boundary disturbance, Internat. J. Robust Nonlinear Control, in press.

[12] B.Z. Guo, Riesz basis approach to the stabilization of a flexible beam with a tip mass, SIAM J. Control Optim., 39(2001), 1736-1747.

[13] J.Q. Han, From PID to active disturbance rejection control, IEEE Transactions on Industrial Electronics, 56(2009), 900-906. 
[14] M. Krstic, B.Z. Guo and A. Smyshlyaev, Boundary controllers and observers for the linearized Schrödinger equation, SIAM J. Control Optim., 49(2011), 1479-1497.

[15] M. Krstic, B.Z. Guo, A. Balogh and A. Smyshlyaev, Output-feedback stabilization of an unstable wave equation, Automatica, 44(2008),63-74.

[16] M. Krstic, Adaptive control of an anti-stable wave PDE, Dyn. Contin. Discrete Impuls. Syst. Ser. A Math. Anal., 17(2010), 853-882.

[17] M. Krstic and A. Smyshlyaev, Boundary Control of PDEs: A Course on Backstepping Designs, Philadelphia, PA: SIAM, 2008.

[18] V. Komornik and P. Loreti, Fourier Series in Control Theory, Springer-Verlag, New York, 2005.

[19] W.J. Liu, Elementary Feedback Stabilization of the Linear Reaction-Convection-Diffusion Equation and the Wave Equation, Springer-Verlag, New York, 2009.

[20] Z.H. Luo, B.Z. Guo and O. Morgul, Stability and Stabilization of Infinite Dimensional Systems with Applications, Springer-Verlag, London, 1999.

[21] E. Machtyngier, Exact controllability for the Schrödinger equation, SIAM J. Control Optim., 32(1994), 24-34.

[22] A. Medvedev and G. Hillerström, An external model control system, Control-Theory and Advanced Technology, 10(1995), 1643-1665.

[23] Y.V. Orlov and V.I. Utkin, Sliding mode control in infinite-dimensional systems, Automatica, 23(1987), 753-757.

[24] A. Pazy, Semigroups of Linear Operators and Applications to Partial Differential Equations, Springer-Verlag, New York, 1983.

[25] A. Pisano, Y. Orlov and E. Usai, Tracking control of the uncertain heat and wave equation via power-fractional and sliding-mode techniques, SIAM J.Control Optim., 49(2011), 363-382.

[26] A. Pisano and Y. Orlov, Boundary second-order sliding-mode control of an uncertain heat process with unbounded matched perturbation, Automatica, 48(2012), 1768-1775.

[27] A. Smyshlyaev and M. Krstic, Closed-form boundary state feedbacks for a class of 1-D partial integro-differential equations, IEEE Trans. Automat. Control, 49(2004), 2185-2202.

[28] J. Vincent, D. Morris, N. Usher, Z. Gao, S. Zhao, A. Nicoletti and Q. Zheng, On active disturbance rejection based control design for superconducting RF cavities, Nuclear Instruments and Methods in Physics Research A, 643(2011), 11-16. 
[29] G. Weiss, Admissible observation operators for linear semigroups, Israel J. Math., 65(1989), 17-43.

[30] G. Weiss, Admissibility of unbounded control operators, SIAM J. Control Optim., 27(1989), 527-545. 